\title{
The Antimedian Function on Paths
}

\author{
Oscar Ortega, Yue Wang \\ Department of Mathematics, Harold Washington College, Chicago, IL, USA \\ Email: oortega@ccc.edu, yue.yale.wang@hotmail.com
}

Received 20 May 2014; revised 19 June 2014; accepted 15 July 2014

Copyright (C) 2014 by authors and Scientific Research Publishing Inc.

This work is licensed under the Creative Commons Attribution International License (CC BY).

http://creativecommons.org/licenses/by/4.0/

(c) (i) Open Access

\begin{abstract}
An antimedian of a sequence $\pi=\left(x_{1}, x_{2}, \cdots, x_{k}\right)$ of elements of a finite metric space $(X, d)$ is an element $x$ for which $\sum_{i=1}^{k} d\left(x, x_{1}\right)$ is a maximum. The function with domain the set of all finite sequences on $X$, and defined by $A M(\pi)=\{x: x$ is an antimedian of $\pi\}$ is called the antimedian function on $X$. In this note, the antimedian function on finite paths is axiomatically characterized.
\end{abstract}

\section{Keywords}

Status, Location Function, Antimedian, Antimedian Function

\section{Introduction}

The problem of finding one optimal location for schools, drug stores, police stations, and hospitals requires facilities to be placed near the users in order to minimize, for example, the distance traveled to reach them. Location theory deals with this type of optimization problem. Location functions such as the median, the center, and the mean have been used to solve these type of problems. On the other hand, there are circumstances where placing one or more facilities as far as possible from the users is the best solution. For instance, it is necessary to locate nuclear power plants far from cities or towns to minimize the risk of radiation problems. Similar problems include the determination of suitable locations for observatories, radio stations, airports, and chemical plants. The solution to the problem of finding an optimal location for these types of obnoxious facilities on networks has been studied by Church and Garfinkel [1], Minieka [2], Ting [3], and Zelinka [4]. In these investigations two solutions to the problem are given from an algorithmic perspective. The most appealing solution is called the antimedian, the points that maximizes the total distance from the facility to the users. Another solution is the anticenter, the points that maximizes the total distance from facilities to users. For more information about obnoxious facilities the reader is remitted to [5]-[7]. In the case of tree graphs, Ting [3] published a linear algorithm to find the antimedian of a tree, and Zelinka [4] proved that the set of leaves of a tree contains an 
antimedian. This problem can be approached through the axiomatization of location functions. The input of a location function consists of some information with respect to the users of the facilities, and the output is related to the consensus reached based on the given information. The rationality of this process is supported by the fact that location functions must satisfy a number of consensus axioms. The mean function on tree graphs was the first location function studied from the axiomatic point of view by Holzman [8] in the continuous case (in the continuous case a tree contains an infinity number of elements, the edges of the tree are considered to be rectifiable curves, and a profile $\pi$ and its members are allowed to be located anywhere on edges). After that Vohra [9] also characterized the median function in the continuous case; in addition the reader can see [10]. In the discrete case, the center, the median, and the mean function have been characterized axiomatically on trees (see for example [11]-[20]). Not much research has been done with respect to the axiomatic characterization of obnoxious location functions, but recently Balakrishnan et al. [21] published a characterization of the antimedian function on paths. In what follows, we present a different axiomatic characterization, also on paths, of the antimedian function. Ortega and Wang have recently sent for publication an axiomatic characterization of the antimean function on paths. For more information about location theory and axiomatization we refer the reader to the following references [22]-[27].

\section{Preliminaries}

Let $(X, d)$ be a finite metric space and set

$$
X^{*}=\bigcup_{k \geq 1} X^{k}
$$

where $X^{k}=\frac{{ }^{k \text { times }}}{X \times \cdots \times X}$ is the cartesian product of $X$. The elements of $X^{*}$ are called profiles and usually denoted by $\pi=\left(x_{1}, x_{2}, \cdots, x_{k}\right)$. Location theory and consensus theory are related to solve the following problem: Given a collection of $k$ users (voters, customers, clients, etc.) with each user having a preferred location point in $X$, one attempts to find a set of elements of $X$ that satisfy the preferences of the users with respect to some well-defined criteria. Modeling this situation requires the use of a location function on $X$, which is a function $L: X^{*} \rightarrow 2^{X} \backslash\{\varnothing\}$, where $2^{X}$ denotes the set of all subsets of $X$. Three well known examples of location functions are:

a) the center function, denoted by Cen, and defined as

$$
\operatorname{Cen}(\pi)=\{x \in X: e(x, \pi) \text { is minimum }\}
$$

where $e(x, \pi)=\max \left\{d\left(x, x_{1}\right), d\left(x, x_{2}\right), \cdots, d\left(x, x_{k}\right)\right\}$.

b) the median function, denoted by Med, and defined as

$$
\operatorname{Med}(\pi)=\left\{x \in X: S_{\pi}(x) \text { is minimum }\right\}
$$

where $S_{\pi}(x)=\sum_{i=1}^{k} d\left(x, x_{i}\right)$.

c) the mean function, denoted by Mean, and defined as

$$
\operatorname{Mean}(\pi)=\left\{x \in X: S_{\pi}(x) \text { is minimum }\right\}
$$

where $S S_{\pi}(x)=\sum_{i=1}^{k} d^{2}\left(x, x_{i}\right)$.

We are interested in finite metric spaces defined in terms of connected graphs. Let $G=(V, E)$ be a finite connected graph, and let $d$ be the usual distance on $V$, where $d(x, y)$ is the length of a shortest path between $x$ and $y$. It is well known that $(V, d)$ is a metric space, and observe that a profile in a graph $G$ is simply a sequence of vertices where repetitions are allowed. We will investigate some properties of the antimedian function on finite metric spaces defined in terms of a very special type of connected graphs, namely paths.

\section{The Antimedian Function on Paths}

In this section $P$ or $P=(V, E)$ will denote a path of length $p$. We will label the vertices of $P$ as $0,1,2, \cdots, p$ and assume that the order that the vertices have in the path is given by the order of the numbers $0,1,2, \cdots, p$. Hence, $P$ will be represented as 


$$
P=0 \rightarrow 1 \rightarrow 2 \rightarrow \cdots \rightarrow p .
$$

Notice that the set of vertices is $V=\{0,1,2, \cdots, p\}$ and also that vertex 0 is adjacent to vertex 1 , vertex 1 is adjacent to vertex 2 and so on. In the case $p$ has an even number of vertices, we will write $p=2 k+1$. In the case $p$ has an odd number of vertices, we will write $p=2 k$. Let $\pi=\left(x_{1}, x_{2}, \cdots, x_{n}\right)$ be a profile on $P$; for any $x \in V$ we define the status of $x$ with respect to $\pi$ to be the number

$$
S_{\pi}(x)=\sum_{i=1}^{k} d\left(x, x_{i}\right) .
$$

A vertex $x$ is called an antimedian of $\pi$ if

$$
S_{\pi}(y) \leq S_{\pi}(x) \quad \forall y \in V .
$$

The antimedian of $\pi$ is the set

$$
A M(\pi)=\{x \in V \mid x \text { is an antime dian of } \pi\} .
$$

In order to study the antimedian function on $P$, we will divide the paths in two classes. The set of paths that have an odd number of vertices will be called odd paths, and the set of paths with an even number of vertices will be called even paths. Let $\pi=\left(x_{1}, x_{2}, \cdots, x_{n}\right)$ be a profile on $P$, the notation

$w \in \pi$

will indicate that there is $i \in\{1,2, \cdots, n\}$ such that $x_{i}=w$. We also use $[\pi]$ to denote the set of all the different vertices included in $\pi$, and the number of vertices in the profile $\pi$ counting repetitions is denoted by $|\pi|$.

For example consider the profile $\pi=(0,0,1,1,5,0,5,5,1,0,3,3)$ on $P$ with $p=5$. In this case $1 \in \pi$, $[\pi]=\{0,1,3,5\}$, and $|\pi|=12$.

If $\pi=\left(x_{1}, x_{2}, \cdots, x_{n}\right)$ and $\beta=\left(y_{1}, y_{2}, \cdots, y_{m}\right)$ are profiles on $P$, denote by $\alpha=\pi \beta$ the profile

$$
\alpha=\pi \beta=\left(x_{1}, x_{2}, \cdots, x_{n}, y_{1}, y_{2}, \cdots, y_{m}\right) .
$$

The profile $\alpha$ is called the concatenation of $\pi$ and $\beta$. The following result related to the antimedian function has been proved in [21]. then

Lemma 1 Let $\pi=\left(x_{1}, x_{2}, \cdots, x_{n}\right)$ and $\beta=\left(y_{1}, y_{2}, \cdots, y_{m}\right)$ be profiles on $P$. If $A M(\pi) \cap A M(\beta) \neq \varnothing$,

$$
A M(\pi \beta)=A M(\pi) \cap A M(\beta) .
$$

The definition of the antimedian function implies the following characteristic of this function.

Lemma 2 Let $\pi=\left(x_{1}, x_{2}, \cdots, x_{n}\right)$ be a profile on $P$, and let $\sigma$ be any permutation of $\{1,2, \cdots, n\}$. Then

$$
A M(\pi)=A M\left(\pi^{\sigma}\right)
$$

where $\pi^{\sigma}=\left(x_{\sigma(1)}, x_{\sigma(2)}, \cdots, x_{\sigma(n)}\right)$.

The median function on finite tree graphs satisfies the following property that was proved in [13], and will be important in the proof of several results.

Lemma 3 Let $\pi=\left(x_{1}, x_{2}, \cdots, x_{n}\right)$ be a profile on a finite tree $T$. If $m \in \operatorname{Med}(\pi)$ and if

$P\left(m, y_{s}\right)=m y_{1} y_{2} \cdots y_{s}$ is a path contained in $T$ such that $y_{1} \notin \operatorname{Med}(\pi)$, then

$$
S_{\pi}(m)<S_{\pi}\left(y_{1}\right)<S_{\pi}\left(y_{2}\right)<\cdots<S_{\pi}\left(y_{s}\right) .
$$

The property of the median function described by Lemma 3 will be called the increasing status property.

Lemma 4 Let $\pi=\left(x_{1}, x_{2}, \cdots, x_{n}\right)$ be a profile on a path $P$ of length $p$. Then

a) $S_{\pi}(0)<S_{\pi}(p)$ implies $A M(\pi)=\{p\}$,

b) $S_{\pi}(p)<S_{\pi}(0)$ implies $A M(\pi)=\{0\}$.

Proof. Notice first that a path is also a tree; consequently, we can apply to $P$ the increasing status property. We first obtain the set $\operatorname{Med}(\pi)$; if $\operatorname{Med}(\pi)=\{t\}$ for some $0 \leq t \leq p$, then we define the paths

$$
P(t, 0)=t \rightarrow t-1 \rightarrow \cdots \rightarrow 0
$$


and

$$
P(t, p)=t \rightarrow t+1 \rightarrow \cdots \rightarrow p .
$$

By the increasing status property we have

$$
S_{\pi}(t)<S_{\pi}(t-1)<S_{\pi}(t-2)<\cdots<S_{\pi}(0)
$$

and

$$
S_{\pi}(t)<S_{\pi}(t+1)<S_{\pi}(t+2)<\cdots<S_{\pi}(p) .
$$

Observe that

if $S_{\pi}(0)<S_{\pi}(p)$, then $A M(\pi)=\{p\}$, and

if $S_{\pi}(p)<S_{\pi}(0)$, then $A M(\pi)=\{0\}$.

On the other hand, assume $\operatorname{Med}(\pi)=\{t, t+1, t+2, \cdots, t+m\}$. Define the paths

$$
P(t, 0)=t \rightarrow t-1 \rightarrow \cdots \rightarrow 0
$$

and

$$
P(t+m, p)=t+m+1 \rightarrow t+m+2 \rightarrow \cdots \rightarrow p .
$$

By the increasing status property we have

$$
S_{\pi}(t)<S_{\pi}(t-1)<S_{\pi}(t-2)<\cdots<S_{\pi}(0)
$$

and

$$
S_{\pi}(t+m+1)<S_{\pi}(t+m+2)<S_{\pi}(t+m+3)<\cdots<S_{\pi}(p) .
$$

If $S_{\pi}(0)<S_{\pi}(p)$, then $A M(\pi)=\{p\}$, and

if $S_{\pi}(p)<S_{\pi}(0)$, then $A M(\pi)=\{0\}$.

We say that a profile $\pi$ on $P$ is of the form $(0, p)^{2 \times s}$ for some integer $s \geq 1$, if $\pi$ contains exactly $s$ times the vertices 0 and $p$. For example the profile $\pi=(0,0,0, p, p, 0, p, p)$ is of the form $(0, p)^{2 \times 4}$. Profiles $\pi$ of the form $(0, p)^{2 \times s}$ are special for the antimedian functions because $A M(\pi)=\{0,1, \cdots, p\}$.

Lemma 5 Let $\pi$ be a profile the form $(0, p)^{2 \times s}$ for some integer $s \geq 1$ on a path $P$ of length $p$. Then $A M(\pi)=\{0,1, \cdots, p\}$.

Proof. It is well known that if $\beta=(a, b)$ is a profile on a finite tree $T$, the median of $\pi$ consists of all the vertices in the path

$$
P(a, b)=a v_{1} v_{2} \cdots v_{s} b
$$

from $a$ to $b$. This implies that

$$
S_{\beta}(a)=S_{\beta}\left(v_{1}\right)=S_{\beta}\left(v_{2}\right)=\cdots=S_{\beta}\left(v_{s}\right)=S_{\beta}(b) .
$$

Since a path $P$ is also a tree, and if $\beta=(a, b)$, then we have

$$
S_{\beta}(0)=S_{\beta}(1)=S_{\beta}(2)=\cdots=S_{\beta}(p),
$$

and the definition of the antimedian function implies that $A M(\beta)=\{0,1, \cdots, p\}$. Because $\pi$ is of the form $(0, p)^{2 \times s}$, then $|\pi|=2 s$, and we can reorder the vertices of $\pi$ to define the profile

$$
\pi^{\prime}=(0, p, 0, p, \cdots, 0, p) .
$$

By Lemmas 1 and 2 we obtain

$$
A M(\pi)=A M\left(\pi^{\prime}\right)=A M(0, p) \cap A M(0, p) \cap \cdots \cap A M(0, p)=\{0,1, \cdots, p\} .
$$

The next result characterizes profiles $\pi$ on a paths of length $p$ that satisfy the condition $S_{\pi}(0)=S_{\pi}(p)$, and that are not of the form $(0, p)^{2 \times s}$. 
Lemma 6 Let $P$ be a path of length $p$, and let $\pi=\left(x_{1}, x_{2}, \cdots, x_{n}\right)$ be a profile that is not of the form $(0, p)^{2 \times s}$ for some integer $s \geq 1$. If $S_{\pi}(0)=S_{\pi}(p)$, then $A M(\pi)=\{0, p\}$.

Proof. Since $P$ is a tree we can apply the increasing status property. We start by obtaining the set $\operatorname{Med}(\pi)$. If $\operatorname{Med}(\pi)=\{t\}$ for some $0 \leq t \leq p$, then we define the paths

$$
P(t, 0)=t \rightarrow t-1 \rightarrow \cdots \rightarrow 0
$$

and

$$
P(t, p)=t \rightarrow t+1 \rightarrow \cdots \rightarrow p
$$

By the increasing status property we obtain

$$
S_{\pi}(t)<S_{\pi}(t-1)<S_{\pi}(t-2)<\cdots<S_{\pi}(0)
$$

and

$$
S_{\pi}(t)<S_{\pi}(t+1)<S_{\pi}(t+2)<\cdots<S_{\pi}(p)
$$

Observe that:

if $S_{\pi}(0)=S_{\pi}(p)$, then $A M(\pi)=\{0, p\}$. On the other hand, assume $\operatorname{Med}(\pi)=\{t, t+1, t+2, \cdots, t+m\}$. Define the paths

$$
P(t, 0)=t \rightarrow t-1 \rightarrow \cdots \rightarrow 0
$$

and

$$
P(t+m, p)=t+m+1 \rightarrow t+m+2 \rightarrow \cdots \rightarrow p .
$$

By the increasing status property we have

$$
S_{\pi}(t)<S_{\pi}(t-1)<S_{\pi}(t-2)<\cdots<S_{\pi}(0)
$$

and

$$
S_{\pi}(t+m+1)<S_{\pi}(t+m+2)<S_{\pi}(t+m+3)<\cdots<S_{\pi}(p) .
$$

Note that $S_{\pi}(0)=S_{\pi}(p)$ implies $A M(\pi)=\{0, p\}$.

From Lemmas 4,5 , and 6 we obtain the following important result that characterizes the output of the antimedian function on paths of length $p$.

Lemma 7 If $\pi=\left(x_{1}, x_{2}, \cdots, x_{n}\right)$ is a profile on a path $P$ of length $p$, then

$$
A M(\pi) \in\{\{0\},\{p\},\{0, p\},\{0,1, \cdots, p\}\} .
$$

Assume

$$
P=0 \rightarrow 1 \rightarrow 2 \rightarrow \cdots \rightarrow p .
$$

is a path of length $p$. If $\pi=(0,1, \cdots, p)$ and $p=2 k$, then $\operatorname{Cen}(\pi)=\{k\}$. Similarly, if $\pi=(0,1, \cdots, p)$ and $p=2 k+1$, then $\operatorname{Cen}(\pi)=\{k, k+1\}$. Denote by $\left[P_{i \leq k}\right]$ the set of vertices $i \in V$ such that $i \leq k$; similarly we define the set $\left[P_{i \geq k+1}\right]$. Using the sets

$$
\left[P_{i \leq k}\right] \cap[\pi] \quad \text { and } \quad\left[P_{i \geq k+1}\right] \cap[\pi]
$$

we define a partition of the profile $\pi$ as follows: denote by $\pi_{i \leq k}$ the profile such that $\left[\pi_{i \leq k}\right]=\left[P_{i \leq k}\right] \cap[\pi]$ and each vertex in $\left[P_{i \leq k}\right] \cap[\pi]$ is included in the profile $\pi_{i \leq k}$ as many times it appears in $\pi$. In a similar way we define the profile $\pi_{i \geq k+1}$ using the set $\left[P_{i \geq k+1}\right] \cap[\pi]$. Notice that $\pi$ can be seen as the concatenation of profiles $\pi_{i \leq k}$ and $\pi_{i \geq k+1}$, in other words $\pi=\pi_{i \leq k} \pi_{i \geq k+1}$. The following number

$$
\Delta_{\pi}=\sum_{x \in \pi_{i \gtrless k+1}} d(x, k)-\sum_{x \in \pi_{i \leq k}} d(x, k)=S_{\pi_{i \gtrless k+1}}(k)-S_{\pi_{i \leq k}}(k) .
$$

will play an important role in the following sections.

\section{The Antimedian Function on Odd Paths}

In this section 


$$
P=0 \rightarrow 1 \rightarrow 2 \rightarrow \cdots \rightarrow p
$$

represents a path such that $p=2 k$, and note that in this case $\operatorname{Cen}(P)=\{k\}$. Let $\pi=\left(x_{1}, x_{2}, \cdots, x_{n}\right)$ be a profile on $P$; we will use $\pi$ to define a new profile that will be denoted $\pi^{*}$. This profile contains the vertex $k$ repeated $|\pi|$ times. In other words we are assuming that $x_{i}=k$ for all $0 \leq i \leq n$. So, $\pi^{*}$ is the profile

$$
\pi^{*}=(k, k, \cdots, k) \text {. }
$$

We want to establish a relationship between $S_{\pi}(0)$ and $S_{\pi^{*}}(0)$. From the definition of profiles $\pi_{i \leq k}$ and $\pi_{i \geq k+1}$ we derive the identities

$$
d(0, x)=d(0, k)-d(x, k) \quad \forall x \in \pi_{i \leq k} .
$$

and

$$
d(0, x)=d(0, k)+d(x, k) \quad \forall x \in \pi_{i \geq k+1} .
$$

Using (3), (4), and the definitions of $S_{\pi}(0)$ and $S_{\pi^{*}}(0)$, we get

$$
\begin{aligned}
S_{\pi}(0)= & \sum_{x \in \pi_{i \leq k}} d(0, x)+\sum_{x \in \pi_{i \gtrless k+1}} d(0, x)=\sum_{x \in \pi_{i \leq k}}[d(0, k)-d(x, k)]+\sum_{x \in \pi_{i \gtrless k+1}}[d(0, k)+d(x, k)] \\
& =S_{\pi^{*}}(0)-\sum_{x \in \pi_{i \leq k}} d(x, k)+\sum_{x \in \pi_{i \gtrless k+1}} d(x, k)=S_{\pi^{*}}(0)+S_{\pi_{i \gtrless k+1}}(k)-S_{\pi_{i \leq k}}(k) .
\end{aligned}
$$

In terms of $\Delta_{\pi}$, defined by (2), and $S_{\pi^{*}}(0)$ we deduce the following relation for $S_{\pi}(0)$

$$
S_{\pi}(0)=S_{\pi^{*}}(0)+\Delta_{\pi}
$$

The next result is corollary to the definition of the number $\Delta_{\pi}$.

Corollary 1 If $\pi=\left(x_{1}, x_{2}, \cdots, x_{n}\right)$ is a profile on $P$, then $\Delta_{\pi}=0$ if and only if $S_{\pi_{i \geq k+1}}(k)=S_{\pi_{i \leqslant k}}(k)$.

The definition of $\pi_{i \leq k}$ and $\pi_{i \geq k+1}$ implies

$$
\begin{gathered}
d(p, x)=d(p, k)+d(k, x) \quad \forall x \in \pi_{i \leq k} \\
d(p, x)=d(p, k)-d(k, x) \quad \forall x \in \pi_{i \geq k+1} .
\end{gathered}
$$

These relations imply

$$
\begin{aligned}
S_{\pi}(p) & =\sum_{x \in \pi_{i \leq k}} d(p, x)+\sum_{x \in \pi_{i \gtrless k+1}} d(p, x)=\sum_{x \in \pi_{i \leq k}}[d(p, k)+d(k, x)]+\sum_{x \in \pi_{i \gtrless k+1}}[d(p, k)-d(k, x)] \\
& =S_{\pi^{*}}(p)+\sum_{x \in \pi_{i \leq k}} d(k, x)-\sum_{x \in \pi_{i \gtrless k+1}} d(k, x)=S_{\pi^{*}}(p)+S_{\pi_{i \leq k}}(k)-S_{\pi_{i \gtrless k+1}}(k) \\
& =S_{\pi^{*}}(p)-\left(S_{\pi_{i \gtrless k+1}}(k)-S_{\pi_{i \leq k}}(k)\right)=S_{\pi^{*}}(p)-\Delta_{\pi} .
\end{aligned}
$$

The definition of $\pi^{*}$ and the fact that $\operatorname{Cen}(P)=\{k\}$ indicate

$$
S_{\pi^{*}}(0)=S_{\pi^{*}}(p)
$$

The following three lemmas establish an important relationship between the numbers $S_{\pi}(0), S_{\pi}(p)$, and $\Delta_{\pi}$. These results will be used to characterize the antimedian of profiles $\pi$ on $P$.

Lemma 8 If $\pi=\left(x_{1}, x_{2}, \cdots, x_{n}\right)$ is a profile on $P$, then $S_{\pi}(p)=S_{\pi}(0)$ if and only if $\Delta_{\pi}=0$.

Proof. Assume first $S_{\pi}(0)=S_{\pi}(p)$, and notice

$$
\begin{gathered}
S_{\pi}(0)=S_{\pi}(p) \\
S_{\pi^{*}}(0)+\Delta_{\pi}=S_{\pi^{*}}(p)+\Delta_{\pi} \\
\Delta_{\pi}=-\Delta_{\pi} \\
2 \Delta_{\pi}=0
\end{gathered}
$$

This implies $\Delta_{\pi}=0$.

Because of (5) and the fact that $\Delta_{\pi}=0$, we obtain 


$$
\begin{aligned}
\Delta_{\pi} & =-\Delta_{\pi} \\
S_{\pi^{*}}(0)+\Delta_{\pi} & =S_{\pi^{*}}(p)-\Delta_{\pi} \\
S_{\pi}(0) & =S_{\pi}(p)
\end{aligned}
$$

Replacing the equal sign with $<$ and $>$ in the proof of Lemma 8, we obtain the next two results.

Lemma 9 If $\pi=\left(x_{1}, x_{2}, \cdots, x_{n}\right)$ is a profile on $P$, then $S_{\pi}(0)<S_{\pi}(p)$ if and only if $\Delta_{\pi}<0$.

Lemma 10 If $\pi=\left(x_{1}, x_{2}, \cdots, x_{n}\right)$ is a profile on $P$, then $S_{\pi}(0)>S_{\pi}(p)$ if and only if $\Delta_{\pi}>0$.

We end this section with an important result that characterizes the antimedian of a profile $\pi$ on odd paths that is not of the form $(0, p)^{2 \times s}$ for some integer $s \geq 1$.

Lemma 11 Let $\pi=\left(x_{1}, x_{2}, \cdots, x_{n}\right)$ be a profile on an odd path $P$. If $\pi$ is not of the form $(0, p)^{2 \times s}$ for some integer $s \geq 1$, then

$$
A M(\pi)= \begin{cases}\{0, p\}, & \text { if } \Delta_{\pi}=0 ; \\ \{0\}, & \text { if } \Delta_{\pi}>0 ; \\ \{p\}, & \text { if } \Delta_{\pi}<0 .\end{cases}
$$

Proof. Assuming $\Delta_{\pi}=0$ and because $\pi$ is not of the form $(0, p)^{2 \times s}$, then Lemma 8 implies $S_{\pi}(0)=S_{\pi}(p)$, and Lemma 6 proves $A M(\pi)=\{0, p\}$.

If $\Delta_{\pi}>0$, then Lemma 10 shows $S_{\pi}(0)>S_{\pi}(p)$, and Lemma 4 demonstrates $A M(\pi)=\{0\}$.

If $\Delta_{\pi}<0$, then Lemma 9 shows $S_{\pi}(0)<S_{\pi}(p)$, and Lemma 4 proves $A M(\pi)=\{p\}$.

\section{The Antimedian Function on Even Paths}

In this section

$$
P=0 \rightarrow 1 \rightarrow 2 \rightarrow \cdots \rightarrow p
$$

represents a path where $p=2 k+1$; so, we have $k=\frac{p-1}{2}$, and in this case $\operatorname{Cen}(P)=\{k, k+1\}$. Let $\pi$ be a profile on $P$. Using similar ideas as in the last section, we can obtain a relationship between the numbers $S_{\pi}(0), S_{\pi^{*}}(0),|\pi|$, and $\Delta_{\pi}$. Since the profile $\pi_{i \leq k}$ contains all the vertices of $\pi$ whose index is less or equal to $k^{\pi}$, then

$$
d(0, x)=d(0, k)-d(x, k) \quad \forall x \in \pi_{i \leq k} .
$$

Using the profile $\pi_{i \geq k+1}$ we obtain

$$
d(0, x)=d(0, k+1)+d(x, k+1)=d(0, k)+1+d(x, k)-1=d(0, k)+d(x, k) \quad \forall x \in \pi_{i \geq k+1} .
$$

From (6) and (7) and the definition of $S_{\pi}(0)$ and $S_{\pi^{*}}(0)$, we deduce

$$
\begin{aligned}
S_{\pi}(0)= & \sum_{x \in \pi_{i \leq k}} d(0, x)+\sum_{x \in \pi_{i \gtrless k+1}} d(0, x)=\sum_{x \in \pi_{i \leq k}}[d(0, k)-d(x, k)]+\sum_{x \in \pi_{i \gtrless k+1}}[d(0, k)+d(x, k)] \\
& =S_{\pi^{*}}(0)-\sum_{x \in \pi_{i \leq k}} d(x, k)+\sum_{x \in \pi_{i \gtrless k+1}} d(x, k)=S_{\pi^{*}}(0)+S_{\pi_{i \gtrless k+1}}(k)-S_{\pi_{i \leq k}}(k) .
\end{aligned}
$$

In terms of $\Delta_{\pi}$, we have the relation

$$
S_{\pi}(0)=S_{\pi^{*}}(0)+\Delta_{\pi}
$$

Observe that

$$
\begin{array}{ll}
d(p, x)=d(p, k+1)+d(x, k+1)=d(p, k)-1+d(x, k)+1=d(p, k)+d(x, k) & \forall x \in \pi_{i \leq k} . \\
d(p, x)=d(p, k+1)-d(x, k+1)=d(p, k)-1-d(x, k)+1=d(p, k)-d(x, k) & \forall x \in \pi_{i \geq k+1} .
\end{array}
$$

Using a similar argument as above, we obtain 


$$
\begin{aligned}
S_{\pi}(p) & =\sum_{x \in \pi_{i \leq k}} d(p, x)+\sum_{x \in \pi_{i \gtrless k+1}} d(p, x)=\sum_{x \in \pi_{i \leq k}}[d(p, k)+d(k, x)]+\sum_{x \in \pi_{i \gtrless k+1}}[d(p, k)-d(k, x)] \\
& =S_{\pi^{*}}(p)+\sum_{x \in \pi_{i \leq k}} d(k, x)-\sum_{x \in \pi_{i \gtrless k+1}} d(k, x)=S_{\pi^{*}}(p)-\left(S_{\pi_{i \gtrless k+1}}(k)-S_{\pi_{i \leq k}}(k)\right) .=S_{\pi^{*}}(p)-\Delta_{\pi} .
\end{aligned}
$$

The definition of $\pi^{*}$ implies the identity

$$
S_{\pi^{*}}(p)=\sum_{i=0}^{|\pi|}(d(p, k+1)+1)=\sum_{i=0}^{|\pi|}(d(0, k)+1)=S_{\pi^{*}}(0)+|\pi| .
$$

This identity provides the following relation between $S_{\pi}(p)$ and $S_{\pi^{*}}(0)$.

$$
S_{\pi}(p)=S_{\pi^{*}}(0)+|\pi|-\Delta_{\pi} \text {. }
$$

The next three results show some properties of the numbers $S_{\pi}(0), S_{\pi}(p),|\pi|$, and $\Delta_{\pi}$.

Lemma 12 If $\pi=\left(x_{1}, x_{2}, \cdots, x_{n}\right)$ is a profile on $P$, then $S_{\pi}(0)=S_{\pi}(p)$ if and only if $\Delta_{\pi}=\frac{\pi}{2}$.

Proof. Assume first $S_{\pi}(0)=S_{\pi}(p)$, and notice that (8) and (9) indicate

$$
S_{\pi}(0)=S_{\pi}(p)
$$

$$
\begin{gathered}
S_{\pi^{*}}(0)+\Delta_{\pi}=S_{\pi^{*}}(0)+|\pi|-\Delta_{\pi} \\
\Delta_{\pi}=|\pi|-\Delta_{\pi} \\
2 \Delta_{\pi}=|\pi| \\
\Delta_{\pi}=\frac{|\pi|}{2}
\end{gathered}
$$

Conversely, if $\Delta_{\pi}=\frac{|\pi|}{2}$, then

$$
\begin{gathered}
\Delta_{\pi}=\frac{|\pi|}{2} \\
2 \Delta_{\pi}=|\pi| \\
\Delta_{\pi}=|\pi|-\Delta_{\pi} \\
S_{\pi^{*}}(0)+\Delta_{\pi}=S_{\pi^{*}}(0)+|\pi|-\Delta_{\pi} \\
S_{\pi}(0)=S_{\pi}(p)
\end{gathered}
$$

By replacing the equal sign with $<$ and $>$ in the proof of Lemma 12, we obtain the following two results.

Lemma 13 If $\pi=\left(x_{1}, x_{2}, \cdots, x_{n}\right)$ is a profile on $P$, then $S_{\pi}(0)<S_{\pi}(p)$ if and only if $\Delta_{\pi}<\frac{|\pi|}{2}$.

Lemma 14 If $\pi=\left(x_{1}, x_{2}, \cdots, x_{n}\right)$ is a profile on $P$, then $S_{\pi}(0)>S_{\pi}(p)$ if and only if $\Delta_{\pi}>\frac{|\pi|}{2}$.

The next lemma is an important result because it characterizes the antimedian of profiles $\pi$, on even paths, that are not of the form $(0, p)^{2 \times s}$ for some integer $s \geq 1$.

Lemma 15 Let $\pi=\left(x_{1}, x_{2}, \cdots, x_{n}\right)$ be a profile on an even path $P$. If $|\pi| \geq 2$ and $\pi$ is not a profile of the form $(0, p)^{2 \times s}$ for some integer $s \geq 1$, then

$$
A M(\pi)= \begin{cases}\{0, p\}, & \text { if } \Delta_{\pi}=\frac{|\pi|}{2} ; \\ \{0\}, & \text { if } \Delta_{\pi}>\frac{|\pi|}{2} ; \\ \{p\}, & \text { if } \Delta_{\pi}<\frac{|\pi|}{2} .\end{cases}
$$


Proof. Assuming $\Delta_{\pi}=\frac{|\pi|}{2}$ and since $\pi$ is not of the form $(0, p)^{2 \times s}$, Lemma 12 shows $S_{\pi}(0)=S_{\pi}(p)$, and Lemma 6 indicates $A M(\pi)=\{0, p\}$.

If $\Delta_{\pi}>\frac{|\pi|}{2}$, then Lemma 14 implies $S_{\pi}(0)>S_{\pi}(p)$. Now Lemma 4 proves $A M(\pi)=\{0\}$.

If $\Delta_{\pi}<\frac{|\pi|}{2}$, then Lemma 13 shows $S_{\pi}(0)<S_{\pi}(p)$, and Lemma 4 implies $A M(\pi)=\{p\}$.

The next result is a corollary to Lemma 15.

Corollary 2 Let $\pi$ be a profile on $P$. If $\pi$ is of the form $(k, k+1)^{2 \times s}$ for some integer $s \geq 1$, then $A M(\pi)=\{0, p\}$.

Proof. Notice that in this case the profile $\pi_{i \geq k+1}$ contains $s$ times the vertex $k+1$, and the profile $\pi_{i \leq k}$ contains $s$ times the vertex $k$. Consequently, we have

$$
\Delta_{\pi}=S_{\pi_{i \gtrless k+1}}(k)-S_{\pi_{i \leq k}}(k)=S_{\pi_{i \gtrless k+1}}(k)=s d(k, k+1)=s=\frac{|\pi|}{2} .
$$

Finally, Lemma 15 implies $A M(\pi)=\{0, p\}$.

\section{The Axioms and the Main Result}

The axioms listed below are among the desirable properties that a general location function should satisfy, and it is not difficult to verify that the antimedian function satisfies these properties.

Oddness (O): Let $L$ be a location function on a path $P$ of length $p$ with $p=2 k$. Let $\Delta_{\pi}$ be defined as in (2); if $\pi$ is not a profile of the form $(0, p)^{2 \times s}$ for some integer $s \geq 1$, then

$$
L(\pi)= \begin{cases}\{0, p\}, & \text { if } \Delta_{\pi}=0 ; \\ \{0\}, & \text { if } \Delta_{\pi}>0 ; \\ \{p\}, & \text { if } \Delta_{\pi}<0 .\end{cases}
$$

Evenness (E): Let $L$ be a location function on a path $P$ of length $p$ with $p=2 k+1$. Let $\Delta_{\pi}$ be defined as in (2); if $\pi$ is not a profile of the form $(0, p)^{2 \times s}$ for some integer $s \geq 1$, then

$$
L(\pi)= \begin{cases}\{0, p\}, & \text { if } \Delta_{\pi}=\frac{|\pi|}{2} ; \\ \{0\}, & \text { if } \Delta_{\pi}>\frac{|\pi|}{2} \\ \{p\}, & \text { if } \Delta_{\pi}<\frac{|\pi|}{2} .\end{cases}
$$

Consistency (C): Let $L$ be a location function on $P$. If $\pi_{1}=\left(x_{1}, x_{2}, \cdots, x_{k}\right)$ and $\pi_{2}=\left(y_{1}, y_{2}, \cdots, y_{s}\right)$ are profiles and $\pi=\left(x_{1}, x_{2}, \cdots, x_{k}, y_{1}, y_{2}, \cdots, y_{s}\right)$ with $L\left(\pi_{1}\right) \cap L\left(\pi_{2}\right) \neq \varnothing$, then $L(\pi)=L\left(\pi_{1}\right) \cap L\left(\pi_{2}\right)$.

Extremeness (Ex): Let $L$ be a location function, and $\pi$ be a profile on $P$. If $\pi=(0, p)$, then $L(\pi)=\{0,1, \cdots, p\}$.

Generalized Extremeness (G-Ex): Let $L$ be a location function, and let $\pi$ be a profile on $P$. If $\pi$ is of the form $(0, p)^{2 \times s}$ for some integer $s \geq 1$, then $L(\pi)=\{0,1, \cdots, p\}$.

Anonymity (A): For any profile $\pi=\left(x_{1}, x_{2}, \cdots, x_{n}\right)$ on $P$ and any permutation $\sigma$ of $\{1,2, \cdots, n\}$, we have $L(\pi)=L\left(\pi^{\sigma}\right)$, where $\pi^{\sigma}=\left(x_{\sigma(1)}, x_{\sigma(2)}, \cdots, x_{\sigma(n)}\right)$.

Some of these axioms are not independent. For example it is clear that (Ex) is a particular case of (G-Ex) when $s=1$. Next we prove that if a location function satisfies (C) and (Ex), it also satisfies (G-Ex).

Lemma 16 If $L$ is a location function on $P$ that satisfies axioms (C), (A), and $(\mathbf{E x})$, then $L$ satisfies axiom (G-Ex). 
Proof. Let $\pi$ be a profile on $P$ of the form $(0, p)^{2 \times s}$ for some integer $s \geq 1$. Corollary 2 implies $A M(\pi)=\{0,1, \cdots, p\}$. Because of axiom (A), we can reorder the vertices of $\pi$ to obtain a profile $\pi^{\prime}$ of the form $\pi^{\prime}=(0, p, 0, p, \cdots, 0, p)$. We can express $\pi^{\prime}$ as a concatenation of profiles of the form $(0, p)$; in other words $\pi^{\prime}=(0, p)(0, p) \cdots(0, p)$. Since $L$ satisfies axiom (Ex), then $L(0, p)=\{0,1, \cdots, p\}$, and applying axiom (C) we conclude

$$
L(\pi)=L\left(\pi^{\prime}\right)=L(0, p) \cap L(0, p) \cap \cdots \cap L(0, p)=\{0,1, \cdots, p\} .
$$

With the axioms listed above we will give two axiomatic characterizations for the antimedian function. The next theorem contains the first of these characterizations.

Theorem 1 Let $L$ be a location function on $T . L$ is the antimedian function on $P$ if and only if $L$ satisfies axioms (-), (E), (Ex), (C), and (A).

Proof. It is clear that if $L$ is the antimedian function, then $L$ satisfies axioms (O), (E), (Ex), (C), and (A). Assume now $L$ is a location function that satisfies axioms $(\mathbf{O}),(\mathbf{E}),(\mathbf{E x}),(\mathbf{C})$, and $(\mathbf{A})$. To prove that $L$ is the antimedian function we consider three cases.

Case 1. Assume first $\pi$ is a profile of the form $(0, p)^{2 \times s}$ for some integer $s \geq 1$, by Lemma 5 we have $A M(\pi)=\{0,1, \cdots, p\}$. Since $L$ satisfies axioms (C), (A), and (Ex), then Lemma 16 proves $L$ satisfies (G-Ex) which implies $L(\pi)=\{0,1, \cdots, p\}$. It is clear that in this case $A M(\pi)=L(\pi)=\{0,1, \cdots, p\}$.

Case 2. Assume $P$ is a path such that $p=2 k$. Let $\pi$ be a profile on $P$ that is not of the form $(0, p)^{2 \times s}$ for some integer $s \geq 1$. Notice that $\operatorname{Cen}(P)=\{k\}$, and if $\Delta_{\pi}=0$, then Lemma 8 shows $S_{\pi}(0)=S_{\pi}(p)$, and Lemma 6 implies $A M(\pi)=\{0, p\}$. On the other hand, since $L$ satisfies axiom (O) and $\Delta_{\pi}=0$, then $L(\pi)=\{0, p\}$. Therefore, $\Delta_{\pi}=0$ means $L(\pi)=A M(\pi)=\{0, p\}$.

If $\Delta_{\pi}>0$, then Lemma 10 indicates $S_{\pi}(0)>S_{\pi}(p)$, and Lemma 4 proves $A M(\pi)=\{0\}$. Since $L$ satisfies axiom (O) and $\Delta_{\pi}>0$, we obtain $L(\pi)=\{0\}$. From this we conclude that if $\Delta_{\pi}>0$, then $A M(\pi)=L(\pi)=\{0\}$. A similar argument can be used to show that if $\Delta_{\pi}<0$, then $A M(\pi)=L(\pi)=\{p\}$.

Case 3. Assume $P$ is a path such that $p=2 k+1$ which means $\operatorname{Cen}(P)=\{k, k+1\}$, and let $\pi$ be a profile on $P$ that is not of the form $(0, p)^{2 \times s}$ for some integer $s \geq 1$. If $\Delta_{\pi}=\frac{|\pi|}{2}$, then Lemma 12 demonstrates $S_{\pi}(0)=S_{\pi}(p)$, and Lemma 6 proves $A M(\pi)=\{0, p\}$. Since $L$ satisfies axiom (E) and $\Delta_{\pi}=\frac{|\pi|}{2}$, we get $L(\pi)=\{0, p\}$. Therefore, $\Delta_{\pi}=\frac{|\pi|}{2}$ implies $L(\pi)=A M(\pi)=\{0, p\}$.

If $\Delta_{\pi}<\frac{|\pi|}{2}$, then Lemma 13 indicates $S_{\pi}(0)<S_{\pi}(p)$, and Lemma 4 shows $A M(\pi)=\{p\}$. Because $L$ satisfies axiom (E) and $\Delta_{\pi}<\frac{|\pi|}{2}, L(\pi)=\{p\}$. Hence, when $\pi$ is a profile that is not of the form $(0, p)^{2 \times s}$ and $\Delta_{\pi}<\frac{|\pi|}{2}, L(\pi)=A M(\pi)=\{p\}$. A similar argument can be used to show that if $\Delta_{\pi}>\frac{|\pi|}{2}$, then $L(\pi)=A M(\pi)=\{0\}$. Notice that Cases 1, 2, and 3 demonstrate the theorem.

We leave it to the reader to prove that the axioms used in the proof of Theorem 1 are independent. Notice that in the proof of Theorem 1 we needed to use three axioms to establish Case 1 . We want to improve the demonstration of this result using fewer axioms. We achieve this objective using axiom (G-Ex) in the following theorem which is our main result.

Theorem 2 Let $L$ be a location function on $T . L$ is the antimedian function on $P$ if and only if $L$ satisfies axioms (0), (E), and (G-Ex).

Proof. It is clear that if $L$ is the antimedian function, then $L$ satisfies axioms (O), (E), and (G-Ex). Assume now that $L$ is a location function that satisfies axioms $(\mathbf{O}),(\mathbf{E})$, and $(\mathbf{G}-\mathbf{E x})$. To prove that $L$ is the antimedian function we consider three cases.

Case 1. Assume first $\pi$ is a profile of the form $(0, p)^{2 \times s}$, then by Lemma 5 we obtain $A M(\pi)=\{0,1, \cdots, p\}$. Because $L$ satisfies axiom (G-Ex), we have $L(\pi)=\{0,1, \cdots, p\}$. So in this case $L(\pi)=A M(\pi)=\{0,1, \cdots, p\}$.

Case 2. Assume $P$ is a path such that $p=2 k$ which means $\operatorname{Cen}(P)=\{k\}$. Let $\pi$ be a profile on $P$ 
that is not of the form $(0, p)^{2 \times s}$. If $\Delta_{\pi}=0$, then Lemma 8 indicates $S_{\pi}(0)=S_{\pi}(p)$, and Lemma 6 implies $A M(\pi)=\{0, p\}$. Since $L$ satisfies axiom (O) and $\Delta_{\pi}=0, L(\pi)=\{0, p\}$. Therefore, $\Delta_{\pi}=0$ indicates $L(\pi)=A M(\pi)=\{0, p\}$. A similar argument can be employed to show that if $\Delta_{\pi}>0$, then $L(\pi)=A M(\pi)=\{0\}$, and if $\Delta_{\pi}<0$, then $L(\pi)=A M(\pi)=\{p\}$.

Case 3. Assume $P$ is a path such that $p=2 k+1$, and let $\pi$ be a profile on $P$. Notice that $\operatorname{Cen}(P)=\{k, k+1\}$. If $\Delta_{\pi}=\frac{|\pi|}{2}$, then Lemmas 12 implies $S_{\pi}(0)=S_{\pi}(p)$, and Lemma 6 shows $A M(\pi)=\{0, p\}$. Because $L$ satisfies axiom (E), we conclude $L(\pi)=\{0, p\}$. Therefore, $\Delta_{\pi}=\frac{|\pi|}{2}$ implies $L(\pi)=A M(\pi)=\{0, p\}$.

A similarly argument can be used to demonstrate that if $\Delta_{\pi}>\frac{|\pi|}{2}$, then $L(\pi)=A M(\pi)=\{0\}$, and if $\Delta_{\pi}<\frac{|\pi|}{2}$, then $L(\pi)=A M(\pi)=\{p\}$. It is clear that Cases 1, 2, and 3 finish the proof of the theorem.

Notice that the definition of axioms (O), (E), and (G-Ex) indicate that they are independent. So it is not necessary to add a proof for the independence of these three axioms. More research is needed to find an axiomatic characterization of the antimedian function on tree graphs.

\section{References}

[1] Church, R.L. and Garinkel, R.S. (1978) Locating an Obnoxious Facility on a Network. Transportation Science, 12, 107-118. http://dx.doi.org/10.1287/trsc.12.2.107

[2] Minieka, E. (1983) Anti-Centers and Anti-Medians of a Network. Networks, 13, 359-365. http://dx.doi.org/10.1002/net.1027

[3] Ting, S.S. (1984) A Linear-Time Algorithm for Maxisum Facility Location on Tree Networks. Transportation Science, 18, 76-84. http://dx.doi.org/10.1287/trsc.18.1.76

[4] Zelinka, B. (1968) Medians and Peripherians of Trees. Archiv der Mathematik, 4, 87-95.

[5] Burkard, R.E., Dollani, H., Lin, Y. and Rote, G. (2001) The Obnoxious Center Problem on a Tree. SIAM Journal on Discrete Mathematics, 14, 498-509. http://dx.doi.org/10.1137/S0895480198340967

[6] Drezner, Z. and Wesolowsky, G.O. (1985) Location of Multiple Obnoxious Facilities. Transportation Science, 19, 193-202. http://dx.doi.org/10.1287/trsc.19.3.193

[7] Labbé, M. (1990) Location of an Obnoxious Facility on a Network: A Voting Approach. Networks, 20, 197-207. http://dx.doi.org/10.1002/net.3230200206

[8] Holzman, R. (1990) An Axiomatic Approach to Location on Networks. Mathematics of Operations Research, 15, 553-563.

[9] Vohra, R. (1996) An Axiomatic Characterization of Some Location in Trees. European Journal of Operational Research, 90, 78-84. http://dx.doi.org/10.1016/0377-2217(94)00330-0

[10] Foster, D.P. and Vohra, R. (1998) An Axiomatic Characterization of a Class of Location in Tree Networks. Operational Research, 46, 347-354. http://dx.doi.org/10.1287/opre.46.3.347

[11] Barthélemy, J.P. and McMorris, F.R. (1986) The Median Procedure for N-Trees. Journal of Classification, 3, $329-334$. http://dx.doi.org/10.1007/BF01894194

[12] Barthélemy, J.P. and Monjardet, B. (1981) The Median Procedure in Cluster Analysis and Social Choice Theory. Mathematical Social Sciences, 1, 235-268. http://dx.doi.org/10.1016/0165-4896(81)90041-X

[13] Kriston, G. and Ortega, O. (2013) The Median Function on Trees. Discrete Mathematics, Algorithms and Applications, 4.

[14] McMorris, F.R., Mulder, H.M. and Ortega, O. (2010) Axiomatic Characterization of the Mean Function on Trees. Discrete Mathematics, Algorithms and Applications, 2, 313-329.

[15] McMorris, F.R., Mulder, H.M. and Ortega, O. (2012) The lp-Function on Trees. Networks, 60, 94-102.

[16] McMorris, F.R., Mulder, H.M. and Powers, R.C. (2003) The Median Function on Distributive Semilattices. Discrete Applied Mathematics, 127, 319-324. http://dx.doi.org/10.1016/S0166-218X(02)00213-5

[17] McMorris, F.R., Mulder, H.M. and Roberts, F.S. (1998) The Median Procedure on Median Graphs. Discrete Applied Mathematics, 84, 165-181. http://dx.doi.org/10.1016/S0166-218X(98)00003-1

[18] McMorris, F.R., Roberts, F.S. and Wang, C. (2001) The Center Function on Trees. Networks, 38, 84-87. 
http://dx.doi.org/10.1002/net.1027

[19] Mulder, H.M., Pelsmajer, M. and Reid, K.B. (2008) Axiomization of the Center Function on Trees. The Australasian Journal of Combinatorics, 41, 223-226.

[20] Ortega, O. (2008) Concensus and Location: The Mean Function. Ph.D. Disertation, Illinois Institute of Technology, Chicago.

[21] Balakrishnan, K., Changat, M., Mulder, H.H. and Subhamathi, A.R. (2012) Axiomatic Characterization of the Antimedian Function on Paths and Hypercubes. Discrete Mathematics, Algorithms and Applications, 4.

[22] Arrow, K.J., Sen, A.K. and Suzumura, K. (2002) Handbook of Social Choice and Welfare, Volumes 1, North Holland, Amsterdam.

[23] Arrow, K.J., Sen, A.K. and Suzumura, K. (2005) Handbook of Social Choice and Welfare, Volumes 2, North Holland, Amsterdam.

[24] Barthélemy, J.P. and Janowitz, M.F. (1991) A Formal Theory of Consensus. SIAM Journal on Discrete Mathematics, 4, 305-322. http://dx.doi.org/10.1137/0404028

[25] Day, W.H.E. and McMorris, F.R. (2003) Axiomatic Consensus Theory in Group Choice and Biomathematics. Frontiers in Applied Mathematics, SIAM, Philadelphia. http://dx.doi.org/10.1137/1.9780898717501

[26] Axiomatic Characterization of Loaction Functions. In: Kaul, H. and Mulder, H., Eds., Advances in Interdisciplinary Applied Discrete Mathematics, Interdisciplinary Mathematical Sciences, Vol. 11 (World Scientific Publishing, Singapure), 2010, 71-91.

[27] Mirchandani, P.B. and Francis, R.L. (1990) Discrete Location Theory. Wiley, New York. 
Scientific Research Publishing (SCIRP) is one of the largest Open Access journal publishers. It is currently publishing more than 200 open access, online, peer-reviewed journals covering a wide range of academic disciplines. SCIRP serves the worldwide academic communities and contributes to the progress and application of science with its publication.

Other selected journals from SCIRP are listed as below. Submit your manuscript to us via either submit@scirp.org or Online Submission Portal.
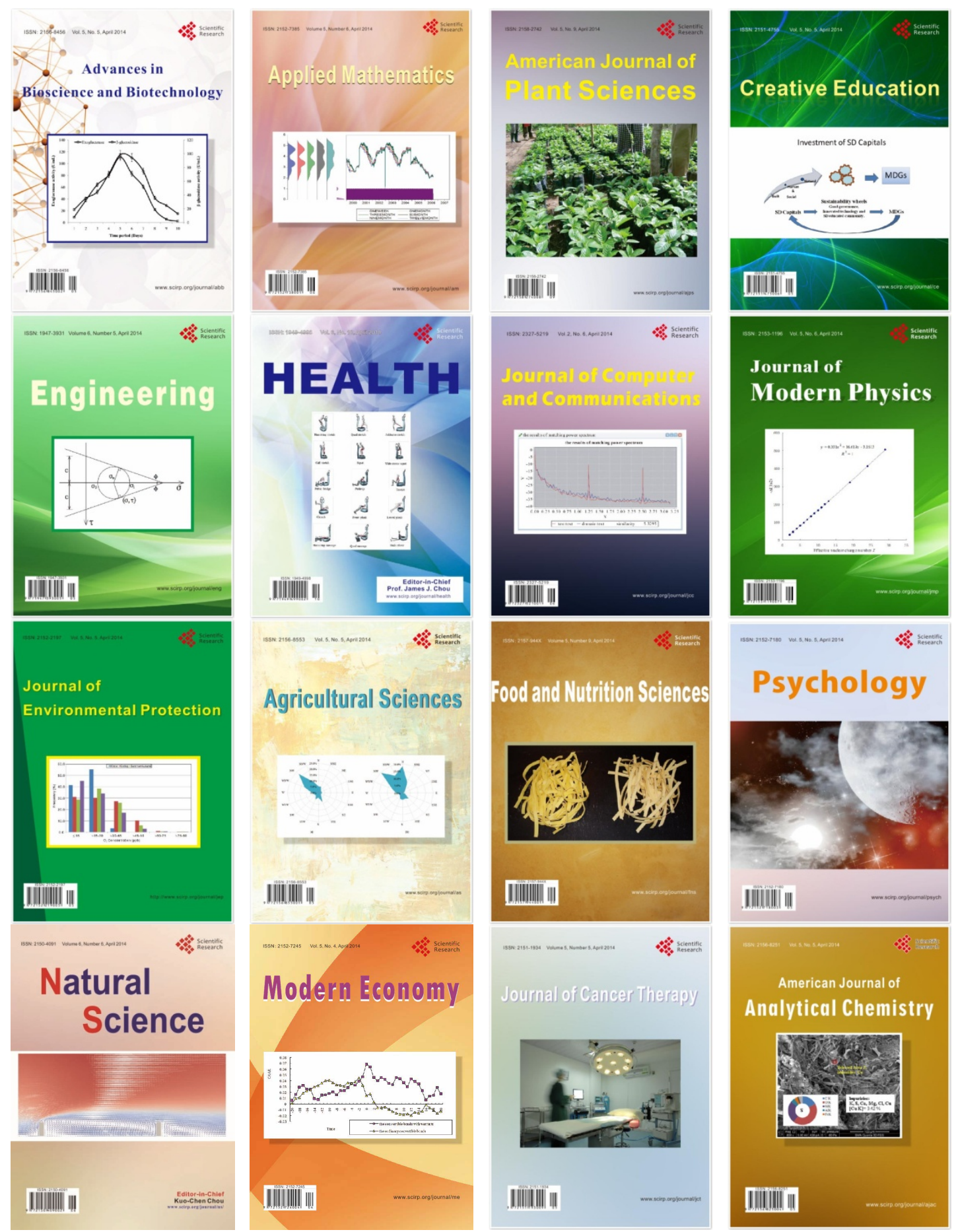